\title{
Intra Parenchymal Extraventricular Supratentorial Ependymomas: Case Report and Review of Pathophysiology and Management
}

\author{
Riadh Rebai ${ }^{\mathrm{a}, \mathrm{b}}$, Khalid AlOrabi ${ }^{\mathrm{a}}$, Ayman Salaheddine ${ }^{\mathrm{a}}$, Saggaf Assagaf ${ }^{\mathrm{a}}$
}

\begin{abstract}
A 6-year-old girl presented with progressive symptoms of increased intracranial pressure of 3 months duration. Computerized Tomography (CT) Scan revealed a right frontal extra-ventricular inhomogeneous space occupying lesion with solid and cystic components including calcifications. She underwent gross total resection of the solid component. Histopathological examination was consistent with ependymoma (WHO grade II). In the post operative course she developed hydrocephalus that was managed by ventriculo-peritoneal shunt insertion. Despite the absence of obvious tumoral residue in post-operative images, the patient was referred for postoperative radiotherapy. Unfortunately, patient was lost of view. Eight months later, she presented to us with headache without focal signs of neurological deficit. MRI (Magnetic resonance imaging) of the brain showed recurrence of the resected frontal tumor. The tumor has been totally removed and patient was referred again to oncology center for adjuvant radiotherapy. Literature was reviewed for pathophysiology, management and prognosis of this rare pathology.
\end{abstract}

Keywords: Supratentorial; Ependymoma; Calcification; Prognosis

\section{Introduction}

Intracranial ependymomas are frequently seen in children and are mainly encountered in the posterior fossa. Considering their cell component, they are usually attached to the ventricles. Supratentorial ependymomas generally affects an older age group (mean age, 18 - 24 years) [1]; only sporadic and rare pediatric cases which were not attached to the ventricle have been reported in the literature. We present a case

\footnotetext{
Manuscript accepted for publication January 22, 2013

${ }^{a}$ King Fahd Hospital (MOH), Department of Neurosurgery, Jeddah, PO box: 50505; Saudi Arabia

${ }^{\mathrm{b}}$ Corresponding author: Riadh Rebai, King Fahd Hospital (MOH), Department of Neurosurgery, Jeddah, PO box: 50505; (int. Box 334) Saudi Arabia.Email: riadh_rebai@yahoo.fr

doi: http://dx.doi.org/10.4021/jmc1062w
}

of a supratentorial intraparenchymal ependymoma in a child reviewing the pathophysiology and discussing the management for such pathology.

\section{Case Report}

A 6-year-old Somali girl patient presented to the emergency department with history of progressive headache and vomiting that started 3 months ago, and was associated with unsteady gait and inability to control urine in the past 3 days. There was no history of convulsions or fever.

On admission, she was drowsy (GCS score $=14 / 15)$, pupils were bilaterally reactive and equal, Papilloedema on fundoscopic examination had left hemiparesis and the cranial nerves were intact. Soon after admission she experienced generalized convulsions. Brain CT scan (Fig. 1A) showed right frontal lobe space-occupying lesion mainly cystic with solid component and calcifications. Both components enhanced with the contrast agent. There was surrounding edema with mass effect on the ipsilateral ventricle and obstructive dilatation of the contra lateral ventricle. MR brain could not be done. She underwent promptly a right frontal craniotomy. Aiming at brain relaxation, the cystic part was primarily tackled through puncturing using a brain cannula and an amount of $30 \mathrm{~mL}$ of brownish fluid was obtained. Corticotomy, until the tumor cyst, exposed the solid component which was red-grayish glistening and multilobulated with highly vascularized and friable structure. The latter was grossly removed. The cystic wall was ill-defined and firmly adherent to parenchyma, so it was spared to avoid brain damage. The lateral ventricle was not opened during the procedure.

Histological examination of the specimen revealed tightly packed cells with oval nuclei having granular chromatin with no evidence of mitosis or atypia. The cells were arranged around the vessels forming perivascular pseudo rosettes with multiple foci of calcification typical of ependymoma (WHO grade II) (Fig. 2A).

Immunohistochemical staining was positive for glial acidic fibrillary protein and epithelial membrane antigen (EMA) (Fig. 2B). 


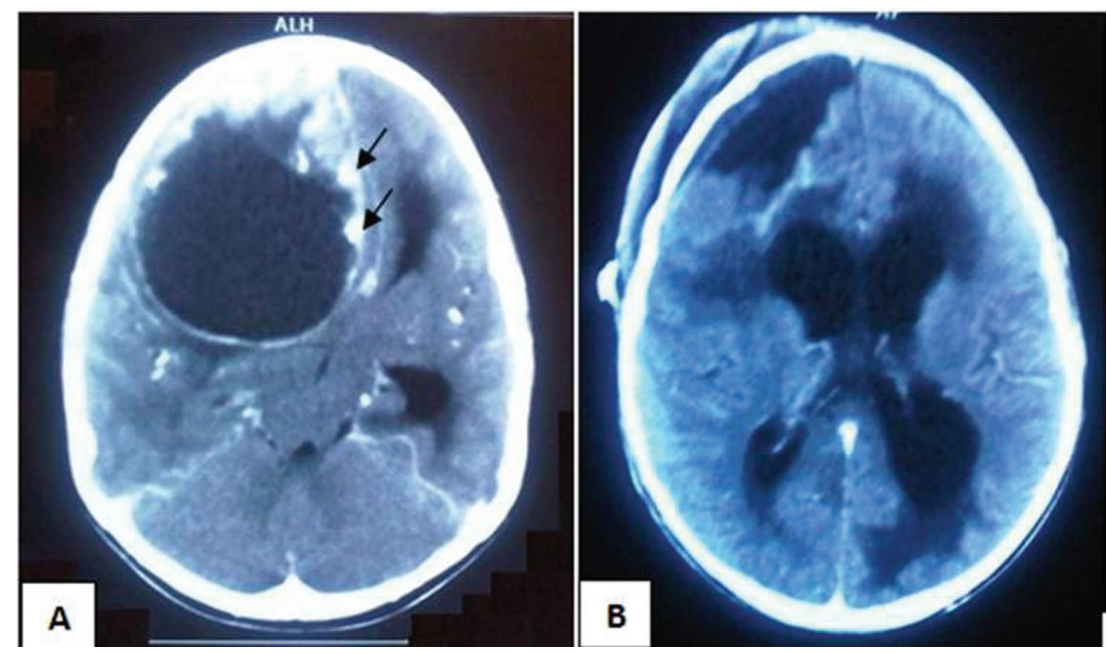

Figure 1. (A): Pre-operative contrasted CT scan depicting huge right frontal lesion including mainly cystic part with solid component along with calcifications (Arrows) and causing obstructive hydrocephalus. (B): Post-operative plain CT scan showing no obvious residual tumor and communicating hydrocephalus.

Postoperative CT scan of the brain (Fig. 1B) showed no tumoral residue but active hydrocephalus managed by insertion of a ventriculo-peritoneal shunt. The post operative course was uneventful. The patient was referred to the oncologist for adjuvant radiotherapy considering the remaining wall of the cystic component. Unfortunately she was lost of view.

Eight months later, the patient presented to us with persistent headache accompanied with nausea. She remained conscious without focal neurological deficit. On enquiry, it appeared that she did not have the recommended adjuvant radiotherapy for socio-economic reason. MRI brain showed recurrence of the right frontal lesion (Fig. 3). The patient underwent surgical revision with total removal of the tumor including the solid component and the cystic one; the right frontal horn was compressed but the ventricle was not invaded. Histopathology of the specimen confirmed the same previous diagnosis with scattered cellular mitosis and mild atypia noted (still grade II). Post operative CT scan did not show any residual tumor (Fig. 3D). The post operative outcome was complicated with meningitis, thus the ventriculoperitoneal shunt was replaced with an external ventricular drainage system with adequate antibiotics prescribed. Patient cured from meningitis and we were able to wean her of the CSF (cerebrospinal fluid) diversion. We thoroughly explained to the family the absolute necessity for completing radiotherapy.

\section{Discussion}

In 1863, Virchow defined ependymomas as tumors with ependymal cells forming ependymal rosettes and perivascular pseudorosettes [2]. They usually develop from the ependy-
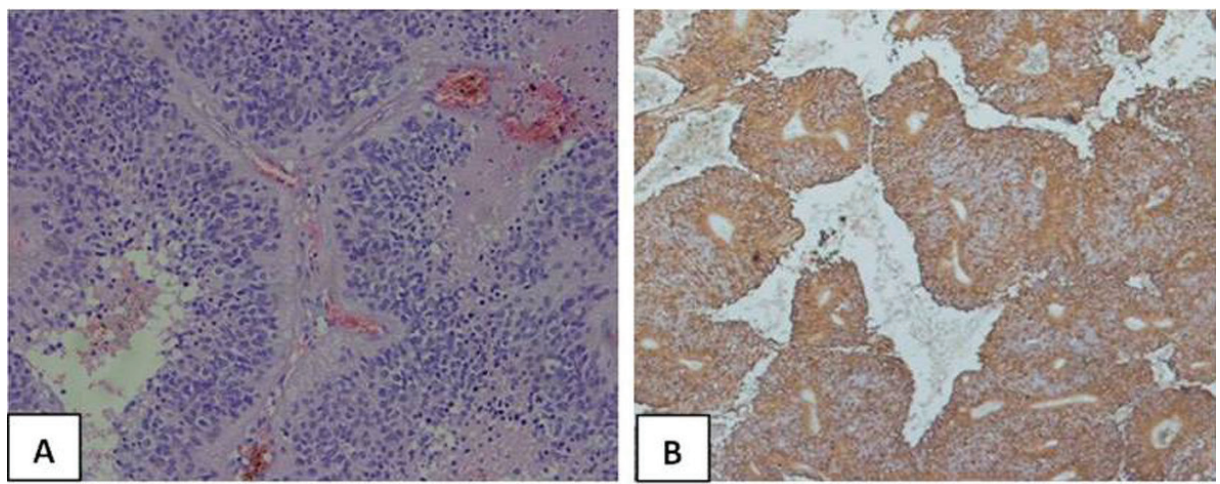

Figure 2. (A): The cytoplasmic processes of ependymal tumor cells condense around blood vessels to form pseudorosettes (Hematoxylin and eosin stain $\times 400)$. (B): Glial fibrillary acidic protein (GFAP) highlights cell processes in perivascular pseudorosettes. (GFAP, $\times 200)$. 


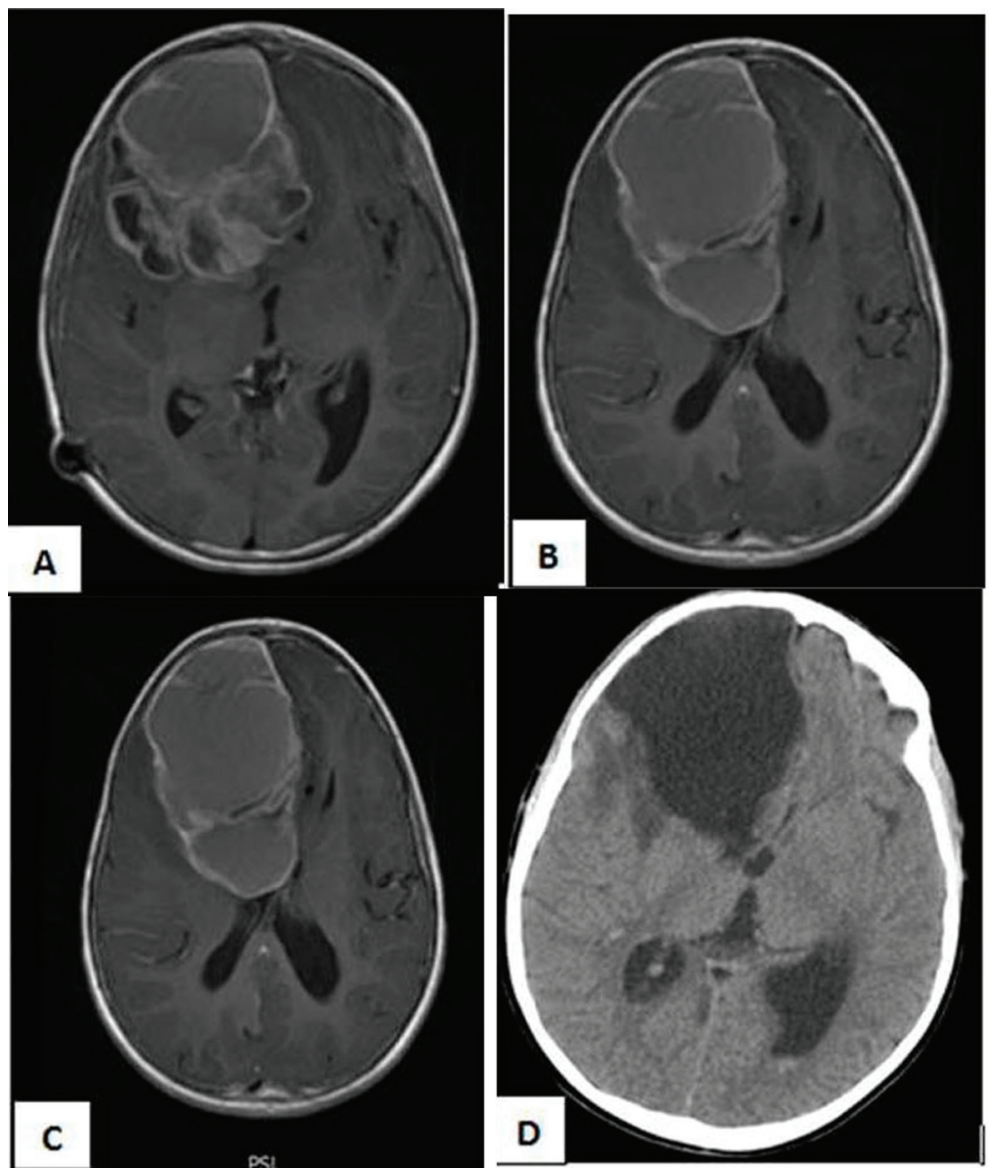

Figure 3. (A, B, C) MRI Brain with Gadolinium, axial cuts showing recurrence of the intracerebral ependymomas with its double enhanced components cystic and solid. (D): Post-operative Ct scan with no residual tumor.

mal and subependymal cells surrounding the cerebral ventricles and the central canal of the spinal cord as well as from ependymal clusters in the filum terminale. Histopathologically, ependymal tumors include low-grade tumors (for example, myxopapillary ependymoma) and high-grade tumors (for example, anaplastic ependymoma) [3]. They comprise approximately 1.2 to $9 \%$ of all brain tumors $[4,5]$. According to the 1993 World Health Organization classification, ependymomas are divided into the following groups: ependymoma, anaplastic (malignant) ependymoma, myxopapillary ependymoma, and subependymoma [3].

\section{Pathophysiology}

Supratentorial ependymomas without relation to the ventricular system are uncommon and their pathophysiology is still not well established.

As its name implies, extra-ventricular ependymomas arise outside the walls of the ventricular system. One of the etiological theories is based on the postulate of remnant nests of ependymal cells in the frontal lobe anterior and inferior to the normal extent of the frontal horns of the lateral ven- tricles in all normal brains; these remnants come from an extension toward the frontal lobe bases of the frontal horns, so-called "olfactory ventricle", that collapses and regresses during normal development. However, random distribution of ependymomas around the periventricular region, rather than restriction to the angles of the ventricles, suggests an alternative mechanism [6].

\section{Diagnostic imaging}

There is no specific MR image for intra parenchymal ependymomas. They may include calcifications in different patterns, or have heterogeneous enhancement. Differential diagnoses include oligodendroglioma, astrocytoma, germ cell tumors, desmoplastic infantile ganglioglioma, and primitive neuroectodermal tumor.

The supratentorial ependymomas tend to be larger in size than those infratentorial. Armington et al [7] found that $94 \%$ of supratentorial tumors manifest with a size larger than $4 \mathrm{~cm}$, while most Infratentorial ependymomas are significantly smaller at presentation. Their shape is irregular in the posterior fossa accommodating to shape of ventricle or cis- 
terns; however they are spherical in the cerebral hemisphere. Supratentorial ependymomas often contain a cystic component, while Infratentorial ependymomas are often more solid tumors [6, 7]. Signal from ependymal cysts is higher than that from CSF. FLAIR sequence better visualizes the border between tumor and ventricular wall. Calcifications, ranging from small punctuate foci to large masses, are very common in both infra and supratentorial ependymomas (40-80\% of cases) $[1,7,8]$. Our patient presented with a tumor larger than $4 \mathrm{~cm}$ in size, mainly cystic with calcifications imbedded in the wall and in the solid part.

An uncommon Intratumoral hemorrhage in supratentorial ependymomas might be mis-interpreted as traumatic in origin following a syncope-head trauma scenario. The tumor will be betrayed by the follow up images showing resorption of the hematoma and clearly depicting the lesion. Takeshima et al. reported a case with at least 3 episodes of hemorrhage before being diagnosed [9]. In our case we anticipate at least one attack of intra-tumoral bleeding prior to presentation based on the perioperative finding of the brownish (altered blood) contents of the cystic component.

\section{Histopathology}

Conversely to the brain infiltrating fibrillary astrocytomas, most extraventricular ependymomas are well circumscribed and well demarcated from the normal cerebral parenchyma thus amenable to total surgical excision. At histological analysis, ependymomas are moderately cellular tumors with rare mitotic figures. The tumor cells are characteristically organized in perivascular pseudorosettes and, less commonly, ependymal rosettes. They are considered World Health Organization (WHO) grade II lesions [1].

Shuangshoti $\mathrm{S}$ et al [10] found that there was no significant relation between histopathology, Ki-67 proliferation index, p53 immunolabeling, tumor ploidy, and biological behavior.

\section{Treatment}

As the tumor is amenable to total radical resection, radical surgery alone is a reasonable option as the initial treatment for solid extraventricular tumors located far from clinically eloquent brain areas. A postoperative MRI with contrast has also been recommended for further evaluation of the extent of resection [11-13]. Early second-look surgery may be proposed to achieve total excision in selected patients with accessible residual tumor detected on postoperative MRI [11].

The need for postoperative adjuvant therapy has been controversial for supratentorial ependymomas. Postoperative radiation therapy must be administered in every case of partially resected ependymomas due to proximity to eloquent areas. So, in general, it is considered safe to observe the patient when postoperative CT or MR shows gross total excision, particularly when the tumor is of low grade. Besides, supratentorial ependymomas tend to recur in regions amenable to surgery. Therefore, reoperation to attempt complete tumor resection before initiation of radiotherapy should be considered $[11,12]$.

It is worth mentioning that some authors recommend adjuvant radiotherapy if the tumor is cystic, even after apparently total resection. Adjuvant radiotherapy has also been suggested to be given to patients with anaplastic ependymomas $[11,12]$. In our case we considered complementary radiotherapy as the cyst wall was incompletely removed after the first surgical procedure.

Prophylactic craniospinal irradiation is no longer advocated unless cerebrospinal seeding is evident on imaging or cerebrospinal fluid studies $[12,14]$. Schild et al reported a series of 80 patients with ependymomas including 12 patients with myxopapillary subtype; in this study the craniospinal irradiation did not have an additional benefit on the survival [15]. Considering the same series, and with a special regard to myxopapillary supratentorial ependymomas which received radiotherapy, the 5-year-local control rate was $100 \%$ with doses exceeding 50 Gy and 67\% with lesser doses [16]. When compared to the other subtypes of ependymomas, patients with myxopapillary ependymomas must be evaluated with serial follow-up because of their propensity to recur locally or metastasize even after several years.

The benefit of chemotherapeutic agents is still questionable. They took place particularly in children for whom radiotherapy is contraindicated or in previously irradiated subjects with an inoperable tumor $[11,17]$.

\section{Prognosis}

Throughout the literature, three major factors were identified determining the outcome. The most important prognostic variable was the presence of radiologic residual disease, seen at postoperative MR imaging or CT. The 5-year-tumorfree survival rate was $75 \%$ and $15 \%$ for patients with no radiologic evidence of residual tumor and those with residual disease in which progression cannot be stopped, respectively [18].

Age at presentation is also a significant prognostic factor (18). Adults have a better five-year survival rate than children. Children less than 2 - 5 years of age, have a significantly worse prognosis than older children; in the young children, five-year survival rate is $22-40 \%$ as compared to $60-75 \%$ in older children [19]. Prognosis in children older than five years is same as that of adults [19]. The 5 and 10year survival rates for adults are $57.1 \%$ and $45 \%$, respectively $[1,20]$.

When considered together, age at diagnosis along with extent of the surgical resection was better correlated to outcome [19].

The last prognostic variable is the duration of symptoms 
preceding diagnosis. Patients with symptoms lasting less than 1 month before diagnosis have a worse outcome than those with a more protracted course [20].

In regards to tumor location, patients with supratentorial ependymomas have generally a better survival rate than patients with posterior fossa ependymomas $[1,21]$.

\section{References}

1. Koeller KK, Sandberg GD. From the archives of the AFIP. Cerebral intraventricular neoplasms: radiologicpathologic correlation. Radiographics. 2002;22(6):14731505.

2. Ho DM, Hsu CY, Wong TT, Chiang H. A clinicopathologic study of 81 patients with ependymomas and proposal of diagnostic criteria for anaplastic ependymoma. J Neurooncol. 2001;54(1):77-85.

3. Kleihues P, Bureger PC, Scheithauer BW, et al: World Health Organization Histological Typing of Tumors of the Central Nervous System. New York, Springer-Verlag, 2nd Ed, p. 17-19, 1993.

4. Lyons MK, Kelly PJ. Posterior fossa ependymomas: report of 30 cases and review of the literature. Neurosurgery. 1991;28(5):659-664; discussion 664-655.

5. Mork SJ, Loken AC. Ependymoma: a follow-up study of 101 cases. Cancer. 1977;40(2):907-915.

6. Furie DM, Provenzale JM. Supratentorial ependymomas and subependymomas: CT and MR appearance. J Comput Assist Tomogr. 1995;19(4):518-526.

7. Armington WG, Osborn AG, Cubberley DA, Harnsberger HR, Boyer R, Naidich TP, Sherry RG. Supratentorial ependymoma: CT appearance. Radiology. 1985;157(2):367372.

8. Morrison G, Sobel DF, Kelley WM, Norman D. Intraventricular mass lesions. Radiology. 1984;153(2):435-442.

9. Takeshima H, Kawahara T, Uchida H, Hirano H, Nakazato Y, Kuratsu J. Brain surface ependymoma with repeated episodes of intratumoral hemorrhage--case report. Neurol Med Chir (Tokyo). 2002;42(4):166-169.

10. Shuangshoti S, Rushing EJ, Mena H, Olsen C, Sandberg
GD. Supratentorial extraventricular ependymal neoplasms: a clinicopathologic study of 32 patients. Cancer. 2005;103(12):2598-2605.

11. Vinchon M, Soto-Ares G, Riffaud L, Ruchoux MM, Dhellemmes P. Supratentorial ependymoma in children. Pediatr Neurosurg. 2001;34(2):77-87.

12. Palma L, Celli P, Mariottini A, Zalaffi A, Schettini G. The importance of surgery in supratentorial ependymomas. Long-term survival in a series of 23 cases. Childs Nerv Syst. 2000;16(3):170-175.

13. Bailey P, Cushing H., et al: A classification of tumors of the glioma group. Philadelphia: Lippincott, 1926.

14. Schwartz TH, Kim S, Glick RS, Bagiella E, Balmaceda C, Fetell MR, Stein BM, et al. Supratentorial ependymomas in adult patients. Neurosurgery. 1999;44(4):721731.

15. Schild SE, Nisi K, Scheithauer BW, Wong WW, Lyons MK, Schomberg PJ, Shaw EG. The results of radiotherapy for ependymomas: the Mayo Clinic experience. Int J Radiat Oncol Biol Phys. 1998;42(5):953-958.

16. Schild SE, Wong W, Nisi K. In regard to the radiotherapy of myxopapillary ependymomas. Int J Radiat Oncol Biol Phys. 2002;53(3):787.

17. Smyth MD, Horn BN, Russo C, Berger MS. Intracranial ependymomas of childhood: current management strategies. Pediatr Neurosurg. 2000;33(3):138-150.

18. Healey EA, Barnes PD, Kupsky WJ, Scott RM, Sallan SE, Black PM, Tarbell NJ. The prognostic significance of postoperative residual tumor in ependymoma. Neurosurgery. 1991;28(5):666-671; discussion 671-662.

19. Spoto GP, Press GA, Hesselink JR, Solomon M. Intracranial ependymoma and subependymoma: MR manifestations. AJR Am J Roentgenol. 1990;154(4):837-845.

20. Pollack IF, Gerszten PC, Martinez AJ, Lo KH, Shultz B, Albright AL, Janosky J, et al. Intracranial ependymomas of childhood: long-term outcome and prognostic factors. Neurosurgery. 1995;37(4):655-666; discussion 666-657.

21. Kudo H, Oi S, Tamaki N, Nishida Y, Matsumoto S. Ependymoma diagnosed in the first year of life in Japan in collaboration with the International Society for Pediatric Neurosurgery. Childs Nerv Syst. 1990;6(7):375-378. 